\title{
Sine-Gordon Parametric Resonance
}

\author{
Patrick B. Greene \\ Department of Physics, University of Toronto, 60 St George Str, Toronto, ON M5S 1A7, Canada* \\ Lev Kofman \\ CITA, University of Toronto, 60 St George Str, Toronto, ON M5S 1A1, Canada ${ }^{\dagger}$ \\ Alexei A. Starobinsky \\ Landau Institute for Theoretical Physics, Kosygina St. 2, Moscow 117334, Russia
}

(September 12, 2018)

\begin{abstract}
We consider the instability of fluctuations in an oscillating scalar field which obeys the Sine-Gordon equation. We present simple closed-form analytic solutions describing the parametric resonance in the Sine-Gordon model. The structure of the resonance differs from that obtained with the Mathieu equation which is usually derived with the small angle approximation to the equation for fluctuations. The results are applied to axion cosmology, where the oscillations of the classical axion field, with a Sine-Gordon self-interaction potential, constitute the cold dark matter of the universe. When the axion misalignment angle at the QCD epoch, $\theta_{0}$, is small, the parametric resonance of the axion fluctuations is not significant. However, in regions of larger $\theta_{0}$ where axion miniclusters would form, the resonance may be important. As a result, axion miniclusters may disintegrate into finer, denser clumps. We also apply the theory of Sine-Gordon parametric resonance to reheating in the Natural Inflation scenario. The decay of the inflaton field due to the self-interaction alone is ineffective, but a coupling to other bosons can lead to preheating in the broad resonance regime. Together with the preheating of fermions, this can alter the reheating scenario for Natural Inflation.
\end{abstract}

PACS: $98.80 . \mathrm{Cq} \quad$ CITA-98-33

\section{INTRODUCTION}

An oscillating pseudo-Nambu-Goldstone (pNG) scalar field, $\phi$, governed by the Sine-Gordon theory and the parametric resonance of its fluctuations, $\phi_{k} e^{i \vec{k} \vec{x}}$, is a prototype for several interesting cosmological and other applications. One of these is the cosmic axion. The axion field, which was originally invented in order to solve the strong CP problem [1], is known to be a dark matter candidate [2, [4], for reviews see e.g. [5] 7]. In the literature, it is often assumed that the axion field is a relatively homogeneous, oscillating classical scalar field which does not decay to other particles. The possibility of a parametric resonance decay of this field due to its self-interaction was considered in Ref. [3]. Using the small angle approximation, which leads to a Mathieu equation, and an elementary estimate for the width of the instability band and the redshifting of the modes due to the expansion of the universe, it was concluded that this process is inefficient. Another important application of the SineGordon theory is to Natural Inflation [8], where after

\footnotetext{
* On leave from the Department of Physics and Astronomy, University of Hawaii, 2505 Correa Rd., Honolulu, HI 96822 USA

${ }^{\dagger}$ On leave from the Institute for Astronomy, University of Hawaii, 2680 Woodlawn Dr., Honolulu, HI 96822, USA
}

inflation a pseudo-Goldstone field oscillates coherently about the minimum of its Sine-Gordon potential. The parametric resonance of inflaton fluctuations due to the self-interaction in the natural inflation scenario was considered in 91. Again, using the small angle approximation and the Mathieu equation, it was concluded that the resonance is not effective. One more application is the resonant decay of disordered chiral condensates [10], modeled with the Sine-Gordon equation [11.

In the last few years, it has been appreciated that parametric resonance of coherently oscillating scalar fields, in particular, the inflaton field (preheating) can be very efficient [12]. For this reason, axion parametric resonance was recently re-examined [13]. The equations governing axion fluctuations were solved numerically in Minkowski space-time and in an expanding universe under the simplifying assumption that the axion mass is a non-vanishing constant since the very beginning of the radiation dominated universe. The results confirmed the conventional wisdom that axion parametric resonance is inefficient because of the expansion of the universe. However, in the cosmic axion scenario, the axion mass is initially absent; it turns on very abruptly at the QCD epoch, $t_{Q C D}$, when instanton effects break the classical Peccei-Quinn symmetry, $U_{P Q}(1)$. After $t_{Q C D}$, the axion begins to oscillate with a period many orders of magnitude smaller than the age of the universe. This is different from the setting of ref. [13], where consequently, the effect of expansion is overestimated. 
In this paper we will systematically study the parametric resonance in the Sine-Gordon theory, both in Minkowski space-time and in an expanding universe. We will show how this problem can be solved analytically and present the theory of the parametric resonance of the Sine-Gordon equation. For this, we will use methods which we elaborated in application to the theory of preheating after inflation 14, 15]. With these novel tools, we will reexamine the axion parametric resonance in an expanding universe where the axion mass is switched on at $t_{Q C D}$. It turns out that the results of the Sine-Gordon parametric resonance can be extended to axions in an expanding universe analytically. In agreement with [3, 13 we will show that the axion parametric resonance is ineffective as soon as the axion misalignment angle at the QCD epoch, $\theta_{0}$, is small, $\theta_{0} \sim 10^{-3}$.

However, there are axion scenarios where the axion field $\theta_{0}$ is spatially inhomogeneous, and in its peak regions can be significantly larger than its mean amplitude. When cosmic axions become gravitationally dominant, these regions will turn into dense axion miniclusters [18,19. We consider the axion resonance in regions where the coherently oscillating axion field of the large amplitude $\theta_{0}$ is smooth at the scales of proto-miniclusters. In these regions, the axion parametric resonance can be quite significant and leads to the decay of the smooth axion field into an inhomogeneous field within protominiclusters. Then, when axions become gravitationally dominant, these inhomogeneities will form small high density clumps inside the minicluster.

Finally, we turn to another application of the SineGordon parametric resonance: reheating in natural inflation. We consider the parametric resonance of inflaton fluctuations due to the self-interaction. We confirm the result of [9], although by different reasoning, that parametric resonance due to the self-interaction is not effective. For the sake of completeness, we also consider the creation of other bosons $\chi$ via a coupling of the form $g^{2} \phi^{2} \chi^{2}$ with the inflaton. In this case, $\chi$-particles can be created in the broad parametric resonance regime; and thus, preheating in natural inflation can be very efficient.

\section{PARAMETRIC RESONANCE IN THE SINE-GORDON THEORY}

First, we neglect expansion of the universe $(a=1$, $H=\frac{\dot{a}}{a}=0$ ), and consider the parametric resonance in the Sine-Gordon theory. We will start with the time evolution of the homogeneous background oscillations in this theory. Then we turn to the basic issue of our study, the stability of small inhomogeneous fluctuations $\phi_{k}(t) e^{i \vec{k} \cdot \vec{x}}$ of comoving momentum $\vec{k}$. Since the background oscillations are periodic, the temporal part of the eigenfunction is $\phi_{k}(t) \sim e^{\mu_{k} m t}$, where the characteristic exponent $\mu_{k}$ is real for resonant modes. The goal of this Section is to find $\mu_{k}$ in the Sine-Gordon theory. Fortunately, one can avoid making any further simplification (besides neglecting expansion) because it is possible to find exact resonance solutions using the methods developed in Ref. [15]. Once we have developed the solutions, we compare the exact result with the estimation obtained from the small angle approximation, $\theta_{0} \ll 1$, which leads to a Mathieu equation for the fluctuations.

\section{A. Background Oscillations in the Sine-Gordon Theory}

The potential of the scalar field in the Sine-Gordon theory can be represented as follows:

$$
V(\phi)=\Lambda^{4}\left(1-\cos \frac{\phi}{f}\right),
$$

where $\Lambda^{4}=m^{2} f^{2}$ with $f$ a constant (corresponding to the radius of the tilted "wine bottle" potential) and $m$ the mass of the bose field $\phi$, see Fig. 1. To simplify notation, we will sometimes use the dimensionless misalignment angle, $\theta \equiv \frac{\phi}{f}$.

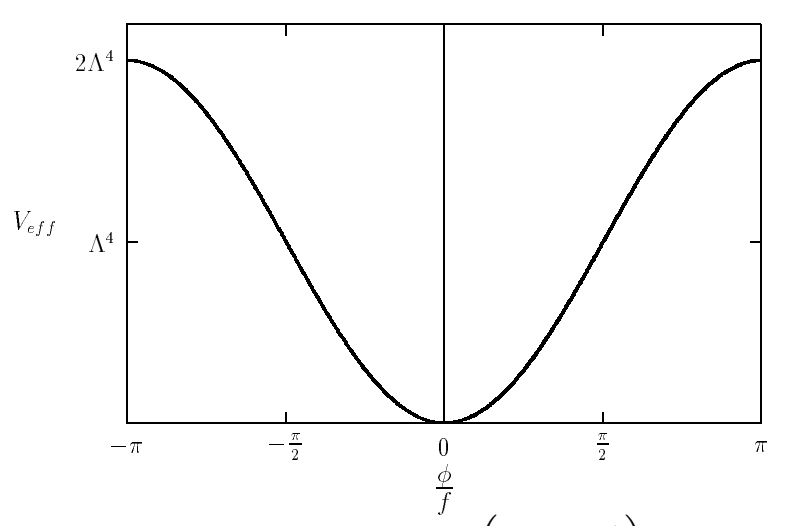

FIG. 1. The potential $\Lambda^{4}\left(1-\cos \frac{\phi}{f}\right)$.

In general, the field $\phi(t, \vec{x})$ obeys the Sine-Gordon equation. For a homogeneous scalar field $\phi=\phi(t)$ and without expansion of the universe, we have

$$
\ddot{\phi}+m^{2} f \sin \frac{\phi}{f}=0,
$$

where $m$ is a constant mass and $f$ is the radius of the potential. For small initial misalignment angle, $\theta_{0} \equiv \frac{\phi_{0}}{f} \ll$ 1 , the solutions to this equation are approximately harmonic. Nevertheless, we will derive the general solution of this equation for an arbitrary initial amplitude without the assumption of a small misalignment angle. Although, for practical purposes in dealing with axion parametric resonance one may skip this general consideration, it is instructive to consider parametric resonance in the SineGordon theory for a wide range of parameters. This will eventually allow us to understand how the parametric 
resonance in an expanding universe can be so sensitive to the initial conditions and parameters involved.

Without loss of generality, we can restrict ourselves to the finite motion $|\phi(t)| \leq \pi f$. The energy integral of Eq. (2) is

$$
\frac{\dot{\phi}^{2}}{2}+V(\phi)=E
$$

where $E \leq 2 m^{2} f^{2}, V(\phi)$ is given by Eq. (1), and $E=$ $V\left(\phi_{0}\right)$.

In Appendix A we derive the final result for the evolution of the background oscillations

$$
\phi(t)=2 f \arctan \left(\sqrt{\frac{\epsilon}{2-\epsilon}} c n(m t ; \mathcal{K})\right),
$$

where the useful dimensionless parameter is $\epsilon \equiv \frac{E}{\Lambda^{4}}=$ $1-\cos \theta_{0}$ and the modulus $\mathcal{K}=\sqrt{\frac{E}{2 \Lambda^{4}}}=\sqrt{\frac{\epsilon}{2}}$. As follows from Eq. (4), it is convenient to use the dimensionless time variable

$$
\tau=m t
$$

As shown in Appendix A, the period of the background fluctuations in the dimensionless time variable, $T_{\tau}$, can be expressed through a complete elliptic integral of the first kind: $T_{\tau}=4 \mathbf{K}\left(\sqrt{\frac{\epsilon}{2}}\right)$. One may check that the amplitude of $\phi(t)$ from Eq. (4) is $\phi_{0}$.

In the small amplitude limit, $\phi_{0} \ll f$, we have $E \approx$ $\frac{m^{2} \phi_{0}^{2}}{2}$ and the oscillations of the background field are approximately harmonic: $\phi(t) \approx \phi_{0} \cos m t, T_{\tau} \approx 2 \pi$.

\section{B. Analytic Resonant Solutions}

We shall consider the fluctuations $\phi_{k}(t)$ in the SineGordon model without expansion of the universe, but not assuming the misalignment angle $\frac{\phi_{0}}{f}$ to be small. In this case the mode equation for fluctuations is

$$
\ddot{\phi}_{k}+\left(k^{2}+m^{2} \cos \frac{\phi}{f}\right) \phi_{k}=0 \text {. }
$$

Let us use the dimensionless time, $\tau=m t$, and the dimensionless momentum $\kappa \equiv \frac{k}{m}$. Then the mode equation is

$$
\ddot{\phi}_{k}+\left(\kappa^{2}+\cos \frac{\phi(\tau)}{f}\right) \phi_{k}=0
$$

where the time-dependence of the background field, $\phi(\tau)$, is given by Eq. (4). Recall that $\phi(\tau)$ is a periodic function of $\tau$ with period $T_{\tau}$.

Equation (7) describes an oscillator, $\phi_{k}(\tau)$, with a variable frequency which depends periodically on time. As is well known, the solutions $\phi_{k}(\tau)$ can be exponentially unstable, $\phi_{k} \propto e^{\mu_{k} \tau}$, for some ranges of the parameters $\kappa$ and $\frac{\phi_{0}}{f}$. For positive-frequency vacuum initial conditions, $\phi_{k}(\tau) \simeq \frac{e^{-i \kappa \tau}}{\sqrt{2 \kappa}}$, one expects the exponentially fast creation of particles $n_{k} \propto e^{2 \mu_{k} \tau}$ in the unstable modes as the background field oscillates. For a given mode, $\kappa$, the strength of interaction is determined by the dimensionless background amplitude, or misalignment angle, $\theta_{0}=\frac{\phi_{0}}{f}$. That is, the absolute value of the misalignment angle, $\theta_{0}$, ultimately defines the structure of the parametric resonance in Sine-Gordon theory. It turns out that the strength of the resonance monotonically depends on this parameter; it is stronger for larger $\left|\theta_{0}\right|$. For $\left|\theta_{0}\right| \geq \pi / 2$ the effective mass is negative, and one has even a strong tachyonic instability.

The main purpose of our study in this Section is to find the characteristic exponent $\mu_{k}=\mu_{k}\left(\theta_{0}\right)$. Even neglecting the expansion of the universe, the equation for fluctuations (7) looks difficult at first glance. Fortunately, it turns out that in the Sine-Gordon theory one can obtain simple, closed-form analytic solutions to the mode equation (7).

For this, let us rewrite this equation in a different form. We will use a new "time" variable $z$ :

$$
z(\tau) \equiv \cos \frac{\phi(\tau)}{f}, \quad \frac{d}{d \tau}=-\sqrt{2\left(1-z^{2}\right)(\epsilon-1+z)} \frac{d}{d z},
$$

where, as before, $\epsilon=\frac{E}{\Lambda^{4}}=1-\cos \theta_{0}$. Note that $z$ is sandwiched in the range $1-\epsilon \leq z \leq 1$. Equation (7) for fluctuations becomes

$$
\begin{aligned}
& 2\left(1-z^{2}\right)(\epsilon-1+z) \phi_{k}^{\prime \prime}+\left(1+2(1-\epsilon) z-3 z^{2}\right) \phi_{k}^{\prime} \\
& +\left(\kappa^{2}+z\right) \phi_{k}=0
\end{aligned}
$$

where $(\ldots)^{\prime}$ stands for the $z$-derivative. As we show in Appendix B, the mode equation in this form coincides with the algebraic form of the Lamé equation with particular coefficients.

At this point we will repeat the trick we found in 15 for a similar problem in $\lambda \phi^{4}$ theory. Specifically, we introduce two linearly-independent solutions of Eq. (9), $\phi_{1}(z)$ and $\phi_{2}(z)$, where the lower index $k$ is omitted for simplicity. Let us construct the bilinear combinations $\phi_{1}^{2}$, $\phi_{2}^{2}$, and $\phi_{1} \phi_{2}$. From (9) it follows that these bilinear combinations obey a third order equation

$$
\begin{aligned}
& 2\left(z^{2}-1\right)(\epsilon-1+z) M^{\prime \prime \prime}+\left[9 z^{2}-6(1-\epsilon) z-3\right] M^{\prime \prime} \\
& +2\left(z-1+\epsilon-2 \kappa^{2}\right) M^{\prime}-2 M=0 .
\end{aligned}
$$

The three solutions, $M(z)$, of this equation correspond to (up to a numerical normalization $\mathcal{N}$ still to be given) the three bilinear combinations of $\phi_{1}$ and $\phi_{2}$.

Equation (10) admits a polynomial solution

$$
M(z)=z-1+\epsilon-2 \kappa^{2} .
$$


In the resonance zone, this polynomial solution must be the product of an exponentially growing solution and an exponentially decreasing one, i.e.

$$
\phi_{1}(z) \phi_{2}(z)=\mathcal{N}^{2} \cdot\left(z-1+\epsilon-2 \kappa^{2}\right),
$$

where $\mathcal{N}$ is a normalization factor. An additional relation between the two fundamental solutions is given by the Wronskian of Eq. (9)

$$
\phi_{1} \phi_{2}^{\prime}-\phi_{2}^{\prime} \phi_{1}=\mathcal{N}^{2} \frac{c_{k}}{\sqrt{\left(1-z^{2}\right)(\epsilon-1+z)}},
$$

where $c_{k}$ is a constant to be determined. From (12) and (13) we obtain the closed form analytic solutions

$$
\begin{aligned}
& \phi_{1,2}(z)=\mathcal{N} \sqrt{|M(z)|} \times \\
& \exp \left( \pm \frac{c_{k}}{2} \int \frac{d z}{\sqrt{\left(1-z^{2}\right)(\epsilon-1+z)} M(z)}\right) .
\end{aligned}
$$

The solution to Eq. (7) with positive-frequency vacuum initial condition will be a linear superposition of the two fundamental solutions $\phi_{1,2} \sqrt{16}$. The amplitude of fluctuations is rapidly dominated by the growing term. As is shown in Appendix $\mathrm{C}$, we can extract the characteristic exponent describing the resonance effect from the fundamental solutions themselves. Therefore we will not further discuss the numerical normalization and, for simplicity, we normalize our fundamental solutions to unit initial amplitude: $\mathcal{N}=|M(1)|^{-1 / 2}$.

\section{The Width and Strength of the Resonance}

The closed-form analytic resonant solution (14) allows us to find the structure of the resonance in terms of the width of the resonance band $\Delta \kappa^{2}$ and its strength $\mu_{k}$ for an arbitrary choice of parameters. Indeed, substituting this solution back into equation (9) for $\phi(z)$, we find the constant $c_{k}$ :

$$
c_{k}^{2}=8 \kappa^{2}\left(\frac{\epsilon}{2}-\kappa^{2}\right)\left(1-\frac{\epsilon}{2}+\kappa^{2}\right) .
$$

For exponentially growing solutions, $c_{k}$ must be real. Therefore, the exponentially growing solutions for fluctuations with $\kappa^{2}>0$ occur in a single instability band for which $0 \leq \kappa^{2} \leq \frac{\epsilon}{2}$. In terms of the basic parameters, the width of the instability band is

$$
0<\kappa^{2}<\frac{1-\cos \theta_{0}}{2}
$$

The growing solution of Eq. (7) has the form $\phi_{k}(\tau)=$ $e^{\mu_{k} \tau} P[z(\tau)]$, where $P[z(\tau)]$ is a periodic function of time $\tau$. Using Eq. (14), we can now find the characteristic exponent $\mu_{k}$ as a function of $\kappa$ for a given initial misalignment angle, $\theta_{0}$. The technical details can be found in Appendix C. The result is

$$
\mu_{k}\left(\theta_{0}\right)=\frac{I_{\kappa}}{T_{\tau}} \sqrt{2 \kappa^{2}\left(1-\left(\cos \theta_{0}+2 \kappa^{2}\right)^{2}\right)},
$$

where an auxiliary function $I_{\kappa}=I_{\kappa}\left(\theta_{0}\right)$ is

$$
\begin{aligned}
& I_{\kappa}\left(\theta_{0}\right)=4 \int_{0}^{\pi / 2} \frac{d \vartheta}{\sqrt{\left(1+\sin ^{2} \vartheta\right)\left(1+\cos \theta_{0} \sin ^{2} \vartheta\right)}} \times \\
& \frac{\sin ^{2} \vartheta}{1+\left(\cos \theta_{0}+2 \kappa^{2}\right) \sin ^{2} \vartheta} \cdot
\end{aligned}
$$

Recall that $T_{\tau}\left(\theta_{0}\right)=4 \mathbf{K}\left(\left|\sin \frac{\theta_{0}}{2}\right|\right)$ is the period of the background oscillations.

In summary, we have found that for an arbitrary value of $\theta_{0}$ there is always a single resonance band given by formula (16). The characteristic exponent strongly depends on the amplitude $\theta_{0}$ and its value is given by an explicit integral in formula (17). The characteristic exponent $\mu_{k}$ found by numerical integration of the equation for fluctuations (7) with vacuum positive-frequency initial conditions is given in Fig. 2 as a function of $\kappa$ for various values of the initial background misalignment angle $\theta_{0}$. These results coincide precisely with the analytic formula (17).

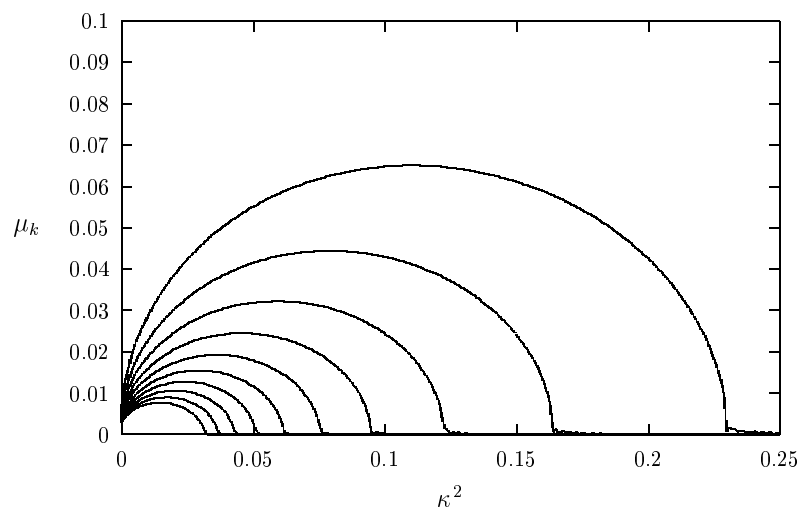

FIG. 2. The characteristic exponent $\mu_{k}$ of the growth of fluctuations $\left(\phi_{k} \propto e^{\mu_{k} \tau}\right)$ as a function of the (scaled) resonant momentum $\kappa$ in Sine-Gordon theory. Different curves correspond to different values of the initial background misalignment angle, $\theta_{0}$. From the outer curve inwards $\theta_{0}=1.67,1.25,1.0,0.83,0.71$, $0.625,0.56,0.5,0.45,0.42,0.38,0.36$.

Let us now consider the case of small misalignment angle $\theta_{0} \ll 1$, which is very important for applications. In the small angle limit, the general formula $(17)$ is reduced to the very simple expression $\mu_{k}=\frac{\kappa}{4} \sqrt{\theta_{0}^{2}-4 \kappa^{2}}$; or explicitly in terms of the momentum $k$,

$$
\mu_{k}=\frac{k}{4 m} \sqrt{\theta_{0}^{2}-\frac{4 k^{2}}{m^{2}}} .
$$

In this case, the maximum value $\mu_{k}=\frac{\theta_{0}^{2}}{16}$ occurs at $\frac{k^{2}}{m^{2}}=$ $\frac{\theta_{0}^{2}}{8}$. The resonance band in this limit is $0 \leq \frac{k^{2}}{m^{2}} \leq \frac{\theta_{0}^{2}}{4}$. In 
the next Section we will extend formula (19) taking into account the expansion of the universe. In this form it will be used to study axion parametric resonance, where the background axion misalignment angle will be small by the QCD time, $t_{Q C D}$.

\section{On the Validity of Use of the Mathieu Equation}

Let us return to the equation for fluctuations (7) and consider a small misalignment angle, $\theta=\frac{\phi}{f} \ll 1$. There we will make the approximation: $\cos \theta(\tau) \approx 1-\frac{1}{2} \theta^{2}(\tau) \approx$ $1-\frac{1}{2} \theta_{o}^{2} \cos ^{2} \tau$. Thus, to this order in $\theta^{2}$, Eq. (汭 becomes

$$
\ddot{\phi}_{k}+\left[\kappa^{2}+\left(1-\frac{\theta_{0}^{2}}{4}\right)-\frac{\theta_{0}^{2}}{4} \cos (2 \tau)\right] \phi_{k}=0,
$$

which is a form of the Mathieu equation, $\ddot{\phi}_{k}+$ $(A-2 q \cos 2 \tau) \phi_{k}=0$, with parameters $A=1-2 q+\kappa^{2}$ and $q=\frac{\theta_{0}^{2}}{8} \ll 1$. The properties of the Mathieu equation for small $q$ parameter are well known 17. The resonance in the first (leading) band occurs for $A=1 \pm q$, which gives a width for the resonance band $\frac{\theta_{0}^{2}}{8} \leq \kappa^{2} \leq \frac{3 \theta_{0}^{2}}{8}$. The characteristic exponent in the first zone is $\mu_{k}^{M}=$ $\sqrt{\left(\frac{q}{2}\right)^{2}-(\sqrt{A}-1)^{2}}$ or, explicitly to this order,

$$
\mu_{k}^{M}=\sqrt{\left(\frac{\theta_{0}^{2}}{16}\right)^{2}-\left(\frac{k^{2}}{2 m^{2}}-\frac{\theta_{0}^{2}}{8}\right)^{2}} .
$$

Note that this expression differs from estimations in [3]. In the Mathieu approximation the leading resonance band is concentrated at $k^{2} \simeq \frac{\theta_{0}^{2}}{4} m^{2}$.

We presented here this analysis because it is simple and intuitive. The approximate solution obtained with the Mathieu equation has some qualitative features similar to the exact solution, but it is quantitatively different. In particular, the actual resonance band begins at $k=0$. Even though this band may be very narrow, the redshifted resonant modes $\phi_{k}$ never shift below this band, i.e. expansion of the universe is not as destructive as one might expect. In this respect, the actual resonance solution is qualitatively different from the approximation based on the Mathieu equation.

\section{BACKGROUND AXION FIELD}

In this and the next section we will investigate axion parametric resonance using the results derived above. Before considering axion fluctuations, we shall discuss the evolution of the background axion field. In the context of the problem of fluctuations, it can be either homogeneous or quasi-homogeneous depending on the cosmic axion scenario and relation of the length scales of fluctuations to the scale where the background field is smooth.
The axion, $\phi$, is a pseudo-Nambu-Goldstone boson which appears after spontaneous symmetry breaking in a theory of a complex scalar field. It acquires a small mass, $m_{a}$, due to instanton effects when the temperature of the universe drops below the QCD scale 22 4]. The effective potential in the simplest theory of the axion field can be represented as (11), where $f$ is the radius of the axion potential [1]. The axion is massless at very high temperature but gradually develops a mass. For $T \gg \Lambda$ we have $m(T) \approx 0.1 m_{a}(\Lambda / T)^{3.7}$. Around the QCD phase transition $(T \approx \Lambda \approx 200 \mathrm{MeV})$, the mass is quickly saturated at its low-temperature value, $m_{a}=\left(\Lambda^{2} / f\right)$, or $m_{a} \approx 0.6 \times 10^{-5}\left(\frac{10^{12} \mathrm{Gev}}{f}\right) \mathrm{eV}$.

At this point, the further discussion of the background axion field branches into different axion scenarios: the initial axion misalignment is globally homogeneous, the initial misalignment is homogeneous within a Hubble domain at the 'defrost' moment $t_{1}$, or the axions are produced by the decay of axionic strings and walls. To investigate the axion parametric resonance due to the selfinteraction of the fluctuations with the background axion oscillations, in principle, one needs to specify the background axion field $\phi(t, \vec{x})$ in these models. However, as we will see below, the axion parametric resonance depends on the value of $\theta_{0}$ at the moment of the QCD phase transition. As soon as a region with given misalignment angle $\theta_{0}$ is smooth and much larger in size than the typical resonant wavelength, one can split the axion field $\phi(t, \vec{x})$ into a background and fluctuations around it.

We first consider the simple model where $\phi(t)$ is a coherent homogeneous field throughout the universe, and then will discuss the inhomogeneous background field $\phi(t, \vec{x})$. A homogeneous axion field obeys the SineGordon equation in an expanding universe

$$
\ddot{\phi}+3 H \dot{\phi}+m^{2}(T) f \sin \frac{\phi}{f}=0 .
$$

The time evolution of the background axion field is plotted in Fig. 3. Before the condition $m(T) \approx 3 H(t)$ holds at a moment $t_{1}$, the axion field is frozen at its initial value $\phi_{1}$ (corresponding to a misalignment angle $\theta_{1}$ ). Shortly after this 'defrost' moment $t_{1}$, the field begins to oscillate with gradually decreasing amplitude. After the axion mass is saturated at $t_{Q C D}$, the coherent fluctuations are approximately harmonic, $\phi(t) \approx \phi_{0} \frac{\sin m_{a} t}{a^{3 / 2}(t)}$, where $\phi_{0}$ is the amplitude at $t_{Q C D}$, and $a=\sqrt{t / t_{Q C D}}$. 


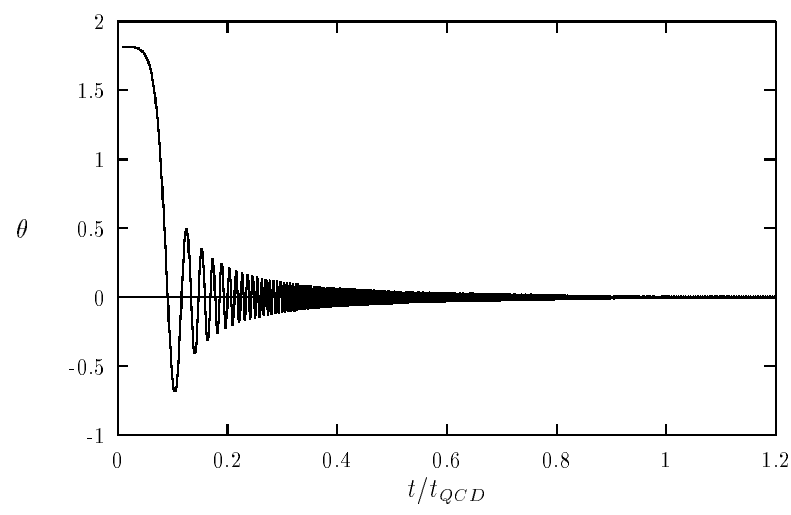

FIG. 3. Time evolution of the background field $\phi(t)$, in terms of the misalignment angle $\theta(t)=\frac{\phi(t)}{f}$, obtained with the numerical solution of Eq. (22). The initial value $\theta_{1}$ is chosen, for illustration, as the r.m.s. of the random phase, i.e. $\theta_{0}=\sqrt{\frac{\pi^{2}}{3}}$.

The resonance regime for axion fluctuations starts at $t_{Q C D}$ (the growth of fluctuations is negligible prior to that moment). Therefore, we have to evaluate the misalignment angle $\theta_{0}$ at $t_{Q C D}$. If the mass density of coherently oscillating axions in units of the critical density is $\Omega_{a}$, then

$$
\theta_{0}^{2} \simeq 10^{-6} \Omega_{a} h^{2}
$$

where $h$ is the present Hubble parameter in units of $100 \mathrm{~km} / \mathrm{s} \cdot$ Mpc. We assume a temperature at $t_{Q C D}$ : $T_{Q C D} \simeq \Lambda_{Q C D} \simeq 200 \mathrm{MeV}$. The value given by Eq. (23) is very small and this, we will see, suppresses the axion resonance.

There are, however, cosmic axion scenarios in which the axion field at the QCD epoch is globally inhomogeneous. In this case, the free axion field $\phi(t, \vec{x})$ obeys the Sine-Gordon equation (22) with an additional gradient term $\frac{1}{a^{2}} \nabla_{\vec{x}}^{2} \phi$. In the uncorrelated misalignment scenario [6, 18 there are rare (overdensity) domains where the initial phase $\theta_{1}$ is as large as $\pi$. As was shown in [18], large inhomogeneities of the background axion field at the present-day scales $t_{1} \times\left(1+z_{1}\right) \simeq 10^{18} \mathrm{~cm}$ upwards result in large density fluctuations by the epoch when axions gravitationally dominate. This leads to the formation of axion miniclusters of the mass $\sim 10^{-12} M_{\odot}$. For our purpose, it will be important that the misalignment angle at $t_{Q C D}$ in the overdensity domains can be significantly larger than the average given by (23). The size, $L$, of these domains, or proto-miniclusters, at $t_{Q C D}$ is given by the size of the horizon at the defrost moment, $t_{1}$, stretched by the scale factor $a\left(t_{Q C D}\right) / a\left(t_{1}\right)$. This size $L$ is smaller than the size of the horizon at $t_{Q C D}$, but significantly larger that the wavelengths $\sim\left(\theta_{0} m_{a}\right)^{-1}$ of axion fluctuations subject to resonant amplification. Therefore, one can consider the resonance of axion fluctuations around a (locally) homogeneous and coherently oscillating field $\theta$ with an amplitude considerably greater than the estimate (23).
The axion field is also inhomogeneous in the scenario where axions are radiated by axionic cosmic strings [20,7]. However, the history of $\theta(t)$ depicted in Fig. 3 is not applicable to this scenario. Again, the mean value of $\theta_{0}$ is given by formula (23), but the axion field at $t_{Q C D}$ is inhomogeneous. Let us briefly discuss the properties of $\theta_{0}(\vec{x})$ in this scenario.

In this scenario, according to Refs. [7], the axion field appears after the universe cools down and PQ symmetry breaking occurs. In different parts of the universe, on the order of the size of the horizon at that moment, the axion field takes different values in the range from 0 to $2 \pi f$. As a result, an axionic string network forms. Starting with the moment $t_{*} \sim 10^{-12}\left(\frac{m_{a}}{10^{-4} \mathrm{eV}}\right)^{4} \mathrm{sec}$, the strings begin to oscillate and radiate their energy into relativistic axions. The dominant contribution comes from the smallest loops at a given time which is some fraction $\alpha$ of the horizon $t, \alpha \sim \kappa$, where $\kappa \simeq 0.15$ is the backreaction scale (in units of $t$ ). The axion spectrum is dominated by the shortest wavelength $\sim \alpha t$. After the axion mass switches on at $t_{1}$, strings transform into domain walls at the moment $t_{w} \approx 1.55 t_{1}$, when further axion emission is terminated. Free axions became nonrelativistic, with a conserved occupation number in each mode. Beginning at the moment $t_{w}$, the net free axion field $\phi(t, \vec{x})$ obeys the Sine-Gordon equation. As initial conditions at $t=t_{w}$, we take $\phi(t, \vec{x})$ to be a superposition of axion waves with uncorrelated phases, emitted by a number of loops, with the spectrum peaked at the mode $k_{w} \sim\left(\alpha t_{w}\right)^{-1}$. In the first approximation, $\phi(t, \vec{x})$ can be modeled by a random Gaussian field with this spectrum. Since we will be interested in the parametric resonance at $t \geq t_{Q C D}$ of the modes $k$ which are many orders of magnitude larger than $k_{w}$, we will model the background field $\phi(t, \vec{x})$ as a quasi-homogeneous field, with the scale of the inhomogeneity $L \sim \alpha t_{w} a\left(t_{Q C D}\right) / a\left(t_{w}\right)$. The peaks of the random field $\theta_{0}(\vec{x})$ can have a field value significantly exceeding the estimation (23). Since $k_{w} \ll m_{a}$, for the epoch $t \geq t_{Q C D}$ we can neglect the gradient term in the Sine-Gordon equation for the background field. At the scales $L$ over which the net axion field $\phi(t, \vec{x})$ can be considered to be homogeneous, it oscillates with the frequency $m_{a}$. This is an approximation to the actual oscillations, which may be neither perfectly coherent nor perfectly harmonic.

\section{AXION PARAMETRIC RESONANCE}

\section{A. Axion Fluctuations}

We represent small fluctuations of the axion field by the modes $\phi_{k}(t) e^{i \vec{k} \cdot \vec{x}}$, where $\vec{k}$ is the comoving momentum. These fluctuations, $\phi_{k}(t)$, obey the equation

$$
\ddot{\phi}_{k}+3 H \dot{\phi}_{k}+\left(\frac{k^{2}}{a^{2}}+m_{a}^{2} \cos \theta\right) \phi_{k}=0 .
$$


One might expect the parametric instability of the fluctuations due to the quasi-periodic variation of the effective frequency in Eq. (24). However, one should also wonder whether the expansion of the universe might suppress this effect 3, 13].

The period of axion oscillations

$\frac{2 \pi}{m_{a}} \approx 8 \times 10^{-10}\left(\frac{5 \cdot 10^{-4} \mathrm{eV}}{m_{a}}\right) \mathrm{s} ;$ meanwhile, the typical time for expansion of the Universe is $H^{-1} \simeq t_{Q C D} \approx$ $7.7 \times 10^{-6} \mathrm{~s}$. Therefore, in the first approximation one can neglect the expansion of the universe in Eq. (24), and calculate the characteristic exponent $\mu_{k}$ for the growth of fluctuations, $\phi_{k} \propto e^{\mu_{k} m_{a} t}$. Then, one can take into account the adiabatic time dependence of $\mu_{k}(t)$ and calculate the net effect as $\phi_{k} \propto \exp \int_{t_{Q C D}} d t \mu_{k}(t) m_{a}$. We will see that this results in a good approximation to the actual evolution as determined by direct numerical solution of the mode equation (24).

\section{B. Resonant Solution with Expansion}

We can now incorporate the expansion of the universe. Fortunately, this also can be done analytically, because the period of axion fluctuations $\sim m_{a}^{-1}$ is much smaller than the Hubble time $H^{-1}$ around $t_{Q C D}$. We incorporate expansion into our static universe results by including the redshifting of the wavelength, $k \rightarrow k / a$, and the dilution of the background field, $\theta_{0}^{2} \rightarrow \theta_{0}^{2} / a^{3}$. Now, instead of expression (19), we have the time-dependent characteristic exponent

$$
\mu_{k}(t)=\frac{k}{4 m_{a} a} \sqrt{\frac{\theta_{0}^{2}}{a^{3}}-\frac{4 k^{2}}{m_{a}^{2} a^{2}}} .
$$

From here it follows that the expansion of the universe can shift the resonant modes out of the resonance. However, as the restructuring of the band via expansion is somewhat faster than the redshifting of modes, the modes actually will be blueshifted relatively to the instability zone. That is, the upper (larger momentum) edge of the instability band will shift toward lower momentum values faster than the modes are redshifted down. From (25) one can immediately estimate the time $t_{k}$ during which a given mode will be amplified, $t_{k}=\left(\frac{\theta_{0} m_{a}}{2 k}\right)^{4} t_{Q C D}$. In Fig. A we plot the evolution of fluctuations as a function of time, obtained by a numerical integration of Eq. (24), which confirms the analytical estimation of $t_{k}$.

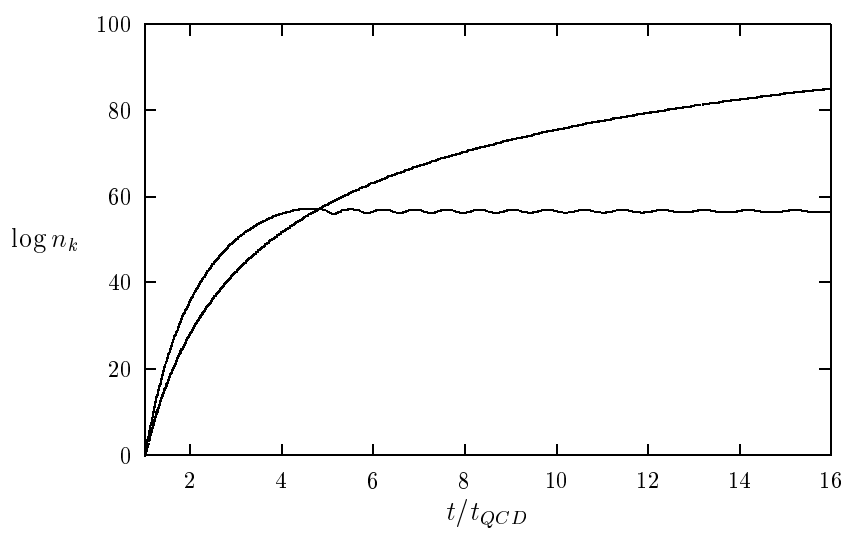

FIG. 4. The log of the axion fluctuations $\left|\phi_{k}\right|^{2}$ in an expanding universe as a function of time (in units of $\left.t_{Q C D}\right)$. We plot the maximally excited mode, $k \approx \frac{1}{5} \theta_{o} m_{a}$ for $\theta_{o}=0.1$ (heavy line) in an expanding universe and the mode $k=\frac{1}{\sqrt{8}} \theta_{o} m_{a}$ that would be maximally excited in a static universe (light line). As discussed in the text, the upper edge of the instability band shifts beneath these modes after a time $t_{k}=\left(\frac{\theta_{o} m_{a}}{2 k}\right)^{4} t_{Q C D}$, which is $42 \times t_{Q C D}$ and $4 \times t_{Q C D}$ for the modes shown.

Amplification of fluctuations by the resonance is determined by the number of e-foldings: $\ln \left|\phi_{k}\right| \simeq$ $\int_{t_{Q C D}}^{t_{k}} d t \mu_{k}(t) m_{a}$, where we have assumed no back reaction for the moment. From Eq. (25) we find

$$
\begin{aligned}
& \int_{t_{Q C D}}^{t_{k}} d t \mu_{k}(t) m_{a} \\
& =\frac{\theta_{0}^{2}}{2 a_{k}}\left(\sqrt{a_{k}-1}-\arctan \sqrt{a_{k}-1}\right)\left(m_{a} t_{Q C D}\right),
\end{aligned}
$$

where, $a_{k}$ is the change in scale factor from $t_{Q C D}$ to $t_{k}$. Specifically,

$$
a_{k}=\sqrt{\frac{t_{k}}{t_{Q C D}}}=\left(\frac{\theta_{0} m_{a}}{2 k}\right)^{2} .
$$

Expression (26) reaches its maximal value

$\int_{t_{Q C D}}^{t_{k}} d t \mu_{k}(t) \approx 0.091\left(m_{a} t_{Q C D}\right) \theta_{0}^{2}$ for $k \approx \frac{1}{5} \theta_{0} m_{a}$. Thus, the leading amplification of the amplitude of a fluctuation is given by

$$
\phi_{k} \simeq 10^{0.039\left(m_{a} t_{Q C D}\right) \theta_{0}^{2}}
$$

This formula is the main technical result of the paper regarding the axionic resonance. The strength of the resonance depends on two numerical factors: the effective number of axion oscillations within a Hubble time around the QCD epoch, $m_{a} t_{Q C D}$, and the value of the axion misalignment angle at $t_{Q C D}, \theta_{0}$. In Fig. 5 we plot the time evolution of the axion fluctuations obtained with the numerical solution of Eq. (24) together with that derived with analytic formula (26) for $\int_{t_{Q C D}}^{t} d t \mu_{k}(t) m_{a}$ for arbitrary $t$. This demonstrates the reliability of our analytic anzats. 


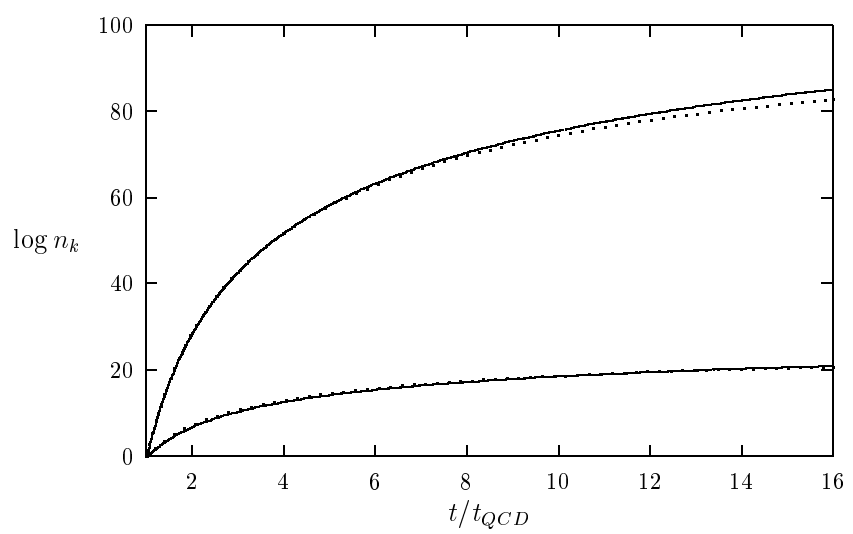

FIG. 5. The $\log$ of the axion fluctuations $\left|\phi_{k}\right|^{2}$ in an expanding universe as a function of time (in units of $t_{Q C D}$ ). We plot the maximally excited mode, $k \approx \frac{1}{5} \theta_{o} m_{a}$, for $\theta_{o}=0.1$ (upper curves) and 0.05 (lower curves). The solid lines are from an exact numerical integration of the mode equation (24) in an expanding universe. The dotted curves are the approximation: $\ln \left|\phi_{k}\right|^{2} \approx 2 \int_{t_{Q C D}}^{t} d t \mu_{k}(t) m_{a}$; the integral is given by formula (26) evaluated for an arbitrary $t>t_{Q C D}$.

\section{Efficiency of the Axion Resonance}

Let us estimate the efficiency of the axion resonance in Eq. (28) for a viable range of parameters. For the axion mass range $m_{a} \simeq 10^{-3}-10^{-4} \mathrm{eV}$ and $t_{Q C D} \simeq 7.7 \times 10^{-6}$ sec, we have $m_{a} t_{Q C D} \approx 5.85 \times 10^{6}\left(\frac{m_{a}}{5 \cdot 10^{-4} \mathrm{eV}}\right)$. Despite this large value for the number of axion oscillations in a Hubble time, the amplification of axion fluctuations is not generally very large because of the small average misalignment angle, $\theta_{0}$, estimated by formula (23). However, as was mentioned at the beginning of this section, in most interesting cases the background field has large scale inhomogeneities. Therefore, we expect to have regions where $\theta_{0}$ exceeds the estimation (23). In this case, only the r.m.s. value of $\theta_{0}$ is given by (23).

To amplify quantum axion fluctuations to the level where fluctuations are comparable with the background field $\left\langle\delta \phi^{2}\right\rangle \sim \phi_{0}^{2}$, the exponent in (28) should be as large as $0.039\left(m_{a} t_{Q C D}\right) \theta_{0}^{2} \gtrsim 23$. Thus, regions of the universe where the background phase exceeds the level

$$
\theta_{0} \gtrsim 10^{-2}\left(\frac{m_{a}}{5 \cdot 10^{-4} \mathrm{eV}}\right)^{-1 / 2}
$$

will have significantly amplified quantum fluctuations of axions. Additionally, it has been noticed [19] that nonlinear axion dynamics prior to $t_{Q C D}$ will further enhance the amplitude in the initially high $\theta_{0}$ regions. If there are small scale, classical axion fluctuations, the threshold value of $\theta_{0}$ for the effective resonance will be even lower. The wavelength of the primary resonant mode, $k \approx \frac{1}{5} \theta_{0} m_{a}$, is significantly smaller that the scale, $L$, over which $\theta_{0}$ is expected to be inhomogeneous

$$
L k \sim 10^{-2} \theta_{0} t_{Q C D} m_{a} \gg 1 .
$$

The region of excessive $\theta_{0}$ corresponds to a protominicluster. The larger $\theta_{0}$ is, the stronger the resonance effect will be. This means that in regions of particularly large $\theta_{0}$, the background field will decay into axion fluctuations within several Hubble times $\sim O(10) t_{Q C D}$. The resulting axion fluctuations can be viewed as a superposition of very slowly moving (non-relativistic) axion waves of wavelength $k^{-1}$. Thus, parametric resonance will cause the (quasi)homogeneous field in the region of a proto-minicluster to become strongly inhomogeneous. Then, when axions come to gravitationally dominant, a large number $\left(\sim(L k)^{3}\right)$ of dense axion clumps will form from the remnants of the proto-minicluster.

We have assumed that the background fluctuations in the region of proto-minicluster are harmonic and coherent. The actual fluctuations may not be perfectly coherent. In this case, one would expect the resonance to be significant if $\theta_{0}$ exceeds a level which is somewhat greater than the idealized estimation (29). To study the resonance in the case when the background fluctuations are not perfectly coherent, one could use lattice simulations like those in [19] but with much greater resolution. Alternatively, one could try to mimic the background fluctuations as a random one dimensional process $\phi(\tau)$. These are, however, beyond the scope of the current paper. Also, we cannot extend our results to the scenarious where axions at $t_{Q C D}$ are relativistic [21]. For now, we consider our results as an indication of possible strong axion resonance in the regions of proto-miniclusters.

\section{PREHEATING IN NATURAL INFLATION}

Another important application of the theory SineGordon parametric resonance elaborated above is to particle production in the Natural Inflation scenario [8], where the pseudo-Nambu-Goldstone boson $\phi(t)$ plays the role of the inflaton field with the potential (11). The typical parameters of Natural Inflation are $f \simeq M_{p}$, $m_{\phi} \simeq 10^{12} \mathrm{GeV}$, and $\Lambda \simeq 10^{15} \mathrm{GeV}$. First, we consider the rate of creation of inflaton quanta due to the selfinteraction. This problem is reduced to the Sine-Gordon parametric resonance in an expanding universe. Then we consider other channels of inflaton decay. Although that issue goes beyond the stability of fluctuations in the SineGordon model, we add this to the paper to complete the picture of reheating and preheating in the Natural Inflation scenario.

\section{A. Self-interacting Inflaton Decay}

The homogeneous inflaton field $\phi(t)$ in this model obeys the equation

$$
\ddot{\phi}+3 H \dot{\phi}+m_{\phi}^{2} f \sin \frac{\phi}{f}=0 .
$$


During the inflationary stage, the field $\phi(t)$ is slowly rolling down to the minimum of the potential (11). After inflation, the field oscillates about the minimum of the potential with a frequency $m_{\phi}$. After several cycles, these oscillations can be simply approximated by harmonic ones, $\phi(t) \approx \Phi(t) \sin m_{\phi} t$, with decreasing amplitude, $\Phi(t)=\frac{\Phi_{0}}{a^{3 / 2}}$. The initial amplitude $\Phi_{0} \simeq O\left(10^{-2}\right) M_{p}$. Inflaton oscillations correspond to the matter dominated equation of state for which $a(t) \propto t^{2 / 3}$.

Let us now turn to the vacuum inflaton fluctuations, $\delta \phi$. The temporal part of an eigenmode, $\phi_{k}(t)$, obeys the equation

$$
\ddot{\phi}_{k}+3 H \dot{\phi}_{k}+\left(\frac{k^{2}}{a^{2}}+m_{\phi}^{2} \cos \theta\right) \phi_{k}=0,
$$

where the oscillating background phase $\theta=\frac{\phi(t)}{f}$ (c.f. Eq. (24)). At the start of oscillations, the typical expansion time, $H^{-1}$, is comparable with the period of oscillations and exceeds it at the latest stages. We find that the parametric resonance of inflaton fluctuations is insignificant. This result can be understood as follows. Resonance occurs in a single resonance band which spans momenta $k / a(t)$ between zero and $\sim \theta_{0}(t) m_{\phi}$, where $\theta_{0}(t)=\frac{\Phi_{0}}{f}=\Theta_{0} a^{-3 / 2}$. For this reason, resonant modes will not be redshifted out of the band, as was suggested in [9]. Just the opposite occurs; momenta redshift more slowly than the background field amplitude, and they become blueshifted relative to the resonance band, just as occurred in the axion case, see Sec. IV B. Let us assume for a moment that the expansion is slow. Then we can use the approximation (25) for the characteristic exponent. The the number of e-foldings $\int d t \mu_{k}(t) m_{\phi}$ will again be proportional to $m_{\phi} t_{0} \theta_{0}^{2}$, where $t_{0}$ is the start of oscillations. However, the factor $m_{\phi} t_{0}$ is of order one, not $10^{6}$ as it was in the axion case. Also, the phase $\theta$ is necessarily rather small. The net result is, at best, a few e-foldings. Now, as the actual expansion at the beginning of the oscillatory phase is not slow, the resonance will be even less efficient. Thus, we confirm the claim of [9] that the parametric resonance of the inflaton field due to the self-interaction in Natural Inflation is insignificant, although our explanation is somewhat different.

\section{B. Resonant Inflation Decay with a $g^{2} \phi^{2} \chi^{2}$ Interaction}

However, to make the picture of reheating in the Natural Inflation scenario more comprehensive, we have to consider other channels of inflaton decay. Let us consider the decay of the inflaton into other bosons $\chi$, via a coupling $\frac{1}{2} g^{2} \phi^{2} \chi^{2}$. Using a decomposition of the Bose field $\chi$ into eigenfunctions $\chi_{k}(t) e^{-i \mathbf{k} \cdot \mathbf{x}}$ with comoving momentum $\mathbf{k}$, we obtain, for their temporal parts,

$$
\ddot{\chi}_{k}+3 H \dot{\chi}_{k}+\left(\frac{k^{2}}{a^{2}}+g^{2} \phi(t)^{2}\right) \phi_{k}=0 .
$$

where the background oscillations $\phi(t)$ are given by the solution of Eq. (31).

Eq. (33) is an oscillator-like equation with a periodic effective frequency. Therefore, we should expect resonant solutions $\chi_{k} \sim \exp \int d t \mu_{k}(t) m_{\phi}$. Indeed, when the amplitude of the background phase is sufficiently low, the background oscillations correspond to those in a massive theory $\frac{1}{2} m_{\phi}^{2} \phi^{2}$. Therefore, Eq. (33) is similar to the wellstudying case of preheating in $\frac{1}{2} m^{2} \phi^{2}$-inflation [14]. We introduce the usual resonance parameter $q=\frac{g^{2} \Phi^{2}}{m^{2}}$. If $q \gg 1$, we will have the creation of $\chi$-particles in the broad resonance regime. However, due to the expansion of the universe there will be no distinct resonance bands, but rather a broad range of resonant momenta $\Delta k \simeq q^{1 / 4} m_{\phi}$ where the resonance occurs in a stochastic manner [14]. The growth of the particle's occupation number $n_{k}(t)$ will be a stochastic process. For moderate values of $q$ we approach the regular resonance regime.

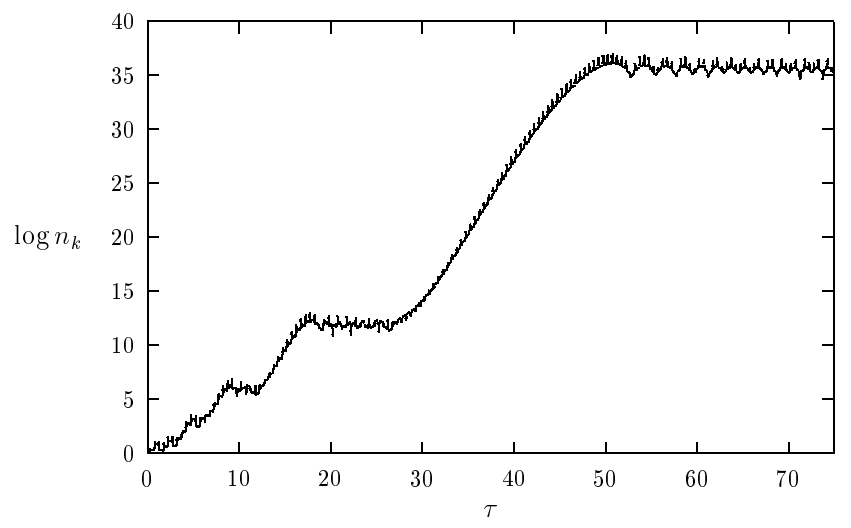

FIG. 6. The typical stochastic resonant production of particles at the particular choice of comoving momentum $k_{0}^{2}=0.1 m_{\phi}^{2}$, and $q=10^{2}$ with expansion. Time is measured in the number of inflaton oscillations since the end of inflation.

Let us estimate the range of allowed $q$ values. From the basic parameters of the Natural Inflation scenario, we have $q=\frac{g^{2} \Phi_{0}^{2}}{m_{\phi}^{2}} \simeq g^{2} 10^{10}$. The coupling parameter $g^{2}$ is restricted by the condition that radiative corrections do not alter the potential (17) $\frac{1}{64 \pi^{2}} g^{4} \phi^{4} \log \frac{\phi}{m} \leq \Lambda^{4}$. This gives us $g \leq \frac{5 \Lambda}{f} \sim 5 \cdot 10^{-4}$, and finally $q \leq 10^{3}$. In Fig. 6 we show the evolution of the number density $n_{k}(t)$ of $\chi$-particles calculated from a numerical solution to the equation for fluctuations (33). Thus, for the resonance parameter $1 \leq q \leq 10^{3}$ the parametric resonance is very effective, and leads to the preheating of bosons $\chi$. The leading channel for inflaton energy transfer will be that with the greatest $q$.

Additionally, it was found very recently that there can be preheating of fermions $\psi$ due to an inflaton coupling $h \bar{\psi} \phi \psi$ [22]. The creation of fermions occurs in the regime of parametric excitation, which is very different from the 
perturbative calculations used earlier [9] for Natural Inflation. In perturbative reheating, the reheat temperature was found to be rather low, $T_{r} \simeq g 10^{8} \mathrm{GeV}$ [8], where $g^{2}$ is coupling between inflatons and other particles. However, as we have seen, the preheating of bosons and fermions in Natural Inflation can be very efficient. Therefore, there it is a possible to have a larger value of the reheating temperature in this scenario than that calculated with the perturbation theory.

\section{SUMMARY}

In this paper, we have discussed the general theory of parametric resonance of fluctuations around an oscillating homogeneous scalar field with a Sine-Gordon potential. We found a closed form analytic resonance solution which shows that, in such a theory, there is a single instability band which starts at zero momentum. The characteristic exponent and width of the resonance band are strongly dependent on the dimensionless amplitude, or misalignment angle, $\theta_{0}$, of the background oscillations, decreasing as $\theta_{0}$ decreases. Although the background oscillations are well described by a massive theory in the regime of small $\theta_{0}$, the correct properties of the parametric resonance in this regime cannot be obtained by making the small angle approximation in the equation for fluctuations, which would lead to a Mathieu equation. This is due to the non-perturbative nature of the parametric resonance.

As a particular application of the general theory of Sine-Gordon parametric resonance, we considered the stability of axion fluctuations in the cosmological model with cosmic axions. By the QCD epoch, axions are non-relativistic and can be considered as a coherently oscillating background field with a misalignment angle $\theta_{0}$ and frequency $m_{a} \sim 10^{-4} \mathrm{eV}$. The expansion of the universe is important to the strength of the resonance, but is not itself deadly as the typical expansion time significantly exceeds the period of axion fluctuations, e.g. $m_{a} t_{Q C D} \gg 1$. If the amplitude of fluctuations is as small as $\theta_{0} \leq 10^{-3}$, as required of the r.m.s. value in an axion dominated cosmology, then the expansion of the universe overdominates for viable ranges of $m_{a}$ and axionic parametric resonance is inefficient. However, there are axion models for which the background field $\theta_{0}(\vec{x})$ at the QCD epoch is not perfectly homogeneous. If there were no resonance, the regions of large $\theta_{0}$ would become axion miniclusters when axions come to gravitationally dominant. Assuming the background oscillations are perfectly coherent in the regions of protominiclusters, we found that the axion fluctuations are unstable in the proto-minicluster regions where $\theta_{0} \gtrsim 10^{-2}$ for $m_{a}=5 \times 10^{-4} \mathrm{eV}$. The wavelengths of the resonant modes are significantly smaller than the expected scale of proto-minicluster regions. Thus, in these regions background axions at rest will resonantly decay into mov- ing non-relativistic axions. In other words, a smoothly varying axion field $\theta_{0}(\vec{x})$ will decay into a strongly inhomogeneous axion field. As a result, at the epoch when axions become gravitationally dominant, instead of a single, massive axion minicluster we will have a minicluster composed of a large number of smaller and denser axion "mini-miniclusters". This may be important for axion detection strategies. The effect of axion resonance in the regions of miniclusters should be checked with lattice simulations or with a more sophisticated analytic treatment of the background oscillations which may not be perfectly coherent. Therefore, we consider our calculations as an indication that there may be strong resonance effects in axion miniclusters.

Another application of the general theory of the SineGordon parametric resonance is to particle production in the Natural Inflation scenario, where the inflaton field has a Sine-Gordon self-interaction. We considered the production of inflaton fluctuations and found that the resonant decay of inflatons is not effective. Beyond the Sine-Gordon self-interaction, we consider the inflaton decay in this scenario via a four-leg interaction with other bosons. The channels of decay for which the coupling parameter is in the region $10^{-5} \leq g \leq 5 \cdot 10^{-4}$ will lead to a broad parametric resonance; smaller $g$ will correspond to a narrow resonance. Together with the preheating of fermions, this opens the possibility of preheating in the Natural Inflation scenario.

\section{ACKNOWLEDGMENTS}

The authors are grateful to A. Linde for valuable discussions and suggestions and to I. Tkachev for useful comments. L.K. was supported in part by NSF grant AST95-29-225. A.S. was supported in part by the Russian Foundation for Basic Research, grant 96-02-17591, and the Russian Research Project "Cosmomicrophysics".

\section{APPENDIX A: BACKGROUND SOLUTION OF THE SINE-GORDON EQUATION}

Here we derive the general homogeneous solution of the Sine-Gordon equation (2) for an arbitrary initial amplitude $\phi_{0}$, not necessarily much smaller than $f$, with no expansion of the Universe taken into account. Without loss of generality, we can restrict ourselves to the finite motion $|\phi(t)| \leq \pi f$, with the constant total energy $E$ given by (3). Let us put $y(t)=\tan \frac{\phi(t)}{2 f}$. Then from the energy integral (3) one finds

$$
\dot{y}^{2}=\frac{1}{2 f^{2}}\left(1+y^{2}\right)\left(E-\left(2 m_{a}^{2} f^{2}-E\right) y^{2}\right) .
$$

Let us now recall the equation for the elliptic cosine, $C \equiv$ $c n(\tau ; \mathcal{K})$, as a function of some variable $\tau$ where $\mathcal{K}$ is its modulus: 


$$
\dot{C}^{2}=\mathcal{K}^{\prime 2}+\left(\mathcal{K}^{2}-\mathcal{K}^{\prime 2}\right) C^{2}-\mathcal{K}^{2} C^{4}, \mathcal{K}^{\prime 2}=1-\mathcal{K}^{2} .
$$

Here, () stands for the $\tau$-derivative. It is easy to check that Eq. (34) can be reduced to an equation for the elliptic cosine by means of the substitutions $y=\sqrt{\frac{E}{2 m_{a}^{2} f^{2}-E}}$ cn $(\tau ; \mathcal{K}), \tau=m_{a} t$ where a dimensionless parameter is $\epsilon=E / m_{a}^{2} f^{2}$ and the modulus is $\mathcal{K}=\sqrt{\epsilon / 2}$. Therefore, for the background oscillations we have $\tan \frac{\phi(t)}{2 f}=\sqrt{\frac{\epsilon}{2-\epsilon}} c n\left(m_{a} t ; \mathcal{K}\right)$, which finally results in Eq. (位). The (dimensionless) period of the background fluctuations, $T_{\tau}$, is equal to

$$
\begin{aligned}
& T_{\tau}=\sqrt{8} \int_{0}^{\arccos (1-\epsilon)} \frac{d \frac{(\phi}{f)}}{\sqrt{\epsilon-1+\cos \frac{(\phi}{f}}} \\
& =\sqrt{8} \int_{0}^{\pi / 2} \frac{d \vartheta}{\sqrt{\left(1+\sin ^{2} \vartheta\right)\left(1+(1-\epsilon) \sin ^{2} \vartheta\right)}},
\end{aligned}
$$

which is equal to $4 \mathbf{K}\left(\sqrt{\frac{\epsilon}{2}}\right)$.

\section{APPENDIX B: ALGEBRAIC FORM OF THE LAMÉ EQUATION}

Here we demonstrate, for the sake of completeness, that the equation for fluctuations $\phi_{k}(t)$ in the SineGordon model (7) (without expansion) can be reduced to the Lamé equation with a particular choice of the coefficients. Indeed, the general algebraic form of the Lamé equation is 23]

$$
\begin{aligned}
& Y^{\prime \prime}+\frac{1}{2}\left(\frac{1}{z-e_{1}}+\frac{1}{z-e_{2}}+\frac{1}{z-e_{3}}\right) Y^{\prime} \\
& +\frac{A z+B}{4\left(z-e_{1}\right)\left(z-e_{2}\right)\left(z-e_{3}\right)} Y=0,
\end{aligned}
$$

where $Y(z)$ is the solution of the Lamé equation. The equation for fluctuations (7) has the form (37) with $e_{1}=$ $1, e_{2}=1-\epsilon, e_{3}=-1, A=-2, n=1, B=\lambda=-2 \kappa^{2}$. Therefore, $\phi_{k}(t)$ is a solution of the Lamé equation with these parameters. Notice that the Lamé equation has already been met in the context of parametric resonance in the theory of preheating with a $\lambda \phi^{4}$ effective potential [15].

\section{APPENDIX C: DERIVATION OF THE CHARACTERISTIC EXPONENT}

Here we show how one can derive Eq. (17) for the characteristic exponent $\mu_{k}$ from the analytic solution (14). Eq. (14) describes both solutions, $\phi_{1}(z)$ and $\phi_{2}(z)$. The resonant solution $\phi(z)$ consists of four monotonic parts within a single period of the background oscillation. It turns out that at different quarters of the period either $\phi_{1}(z)$ or $\phi_{2}(z)$ corresponds to the exponentially growing solution. Indeed, the square of the resonant solution within the first quarter of a period, while $z$ is decreasing from 1 to $(1-\epsilon)$, is

$$
\phi^{2}(z)=\phi_{0}^{2} \exp \left[\int_{1}^{z} \frac{d z}{M(z)}\left(1+\frac{c_{k}}{\sqrt{\left(1-z^{2}\right)(z-1+\epsilon)}}\right)\right],
$$

where $M(z)$ is given by (11), $c_{k}$ is given by (15), and $\phi_{0}^{2}$ is the square of the resonant solution in the beginning of the period when $z=1$.

Within the second quarter of the period, while $z$ is increasing from $(1-\epsilon)$ to 1 , one has

$\phi^{2}(z)=\phi_{1 / 4}^{2} \exp \left[\int_{1-\epsilon}^{z} \frac{d z}{M(z)}\left(1-\frac{c_{k}}{\sqrt{\left(1-z^{2}\right)(z-1+\epsilon)}}\right)\right]$

where $\phi_{1 / 4}$ is the value of $\phi_{z}$ after the first quarter of the period $\phi_{1 / 4} \equiv \phi(z=1)$.

Then the value of $\phi^{2}$ after half of a period is

$$
\phi_{1 / 2}^{2}=\phi_{0}^{2} \exp \left[-2 c_{k} \int_{1-\epsilon}^{1} \frac{d z}{M(z) \sqrt{\left(1-z^{2}\right)(z-1+\epsilon)}}\right]
$$

where the integral is understood as its principal value. The resonant solution has the generic form $\phi(z(\tau))=$ $P(z(\tau)) e^{\mu \tau}$, where $P(z)$ is a periodic function. Since $P$ has a period equal to half of the period of the inflaton oscillation, Eq. (40) is sufficient to find $\mu$ :

$$
\frac{\mu T_{\tau}}{2}=-c_{k} \int_{1-\epsilon}^{1} \frac{d z}{M(z) \sqrt{\left(1-z^{2}\right)(z-1+\epsilon)}}>0 .
$$

The integral in this equation can be reduced to $I\left(\kappa^{2}\right)$ given by (18).

$$
\begin{aligned}
& -\int_{1-\epsilon}^{1} \frac{d z}{M(z) \sqrt{\left(1-z^{2}\right)(z-1+\epsilon)}}= \\
& =2 \int_{0}^{\pi / 2} \frac{d \vartheta}{\sqrt{\left(1+\sin ^{2} \vartheta\right)\left(1+(1-\epsilon) \sin ^{2} \vartheta\right)}} \times \\
& \frac{\sin ^{2} \vartheta}{1+\left(1-\epsilon+2 \kappa^{2}\right) \sin ^{2} \vartheta} \equiv I\left(\kappa^{2}\right) .
\end{aligned}
$$


[1] R.D. Peccei and H.R. Quinn, Phys. Rev. Lett. 38, 1440 (1977); Phys. Rev. D16, 1791 (1977); S. Weinberg, Phys. Rev. Lett. 40, 223 (1978); F. Wilczek, Phys. Rev. Lett. 40, 279 (1978).

[2] J. Preskill, M. Wise and F. Wilczek, Phys. Lett. B120, 127 (1983).

[3] L. Abbott and P. Sikivie, Phys. Lett. B120 133 (1983).

[4] M. Dine and W. Fischler, B120, 137 (1983).

[5] G.G. Raffelt, Phys. Rep. 198, 1 (1990); M.S. Turner, Phys. Rep. 197, 67 (1990); P. Sikivie, hep-ph/9709477.

[6] E. Kolb \& M. Turner, The Early Universe, Addison Wisley, 1990.

[7] R.A. Battye and E.P.S. Shellard, astro-ph/9802216; R.A. Battye and E.P.S. Shellard, Phys. Rev. Lett. 75 (1994) 4354; R.A. Battye and E.P.S. Shellard, Nucl. Phys. B423 (1996) 1811.

[8] K. Freese, J. Frieman, \& A. Olinto, Phys. Rev. Lett. 65 (1990) 3233; F. Adams, J. R. Bond, K. Freese, J. Frieman, \& A. Olinto, Phys. Rev. D 47 (1993) 426

[9] A. Dolgov, K. Freese, Phys. Rev. D51 (1995) 2693

[10] S. Mrowczynski \& B. Muller, Phys. Lett. B363 (1995) 1.

[11] V. Gani, A. Kudryavtsev, T. Belova \& B. Druzhinin, hep-ph/9712526.

[12] L. A. Kofman, A. D. Linde, and A. A. Starobinsky, Phys. Rev. Lett. 73, 3195 (1994)

[13] E. Kolb, A. Singh \& M. Srednicki, hep-ph/970985.

[14] L. A. Kofman, A. D. Linde, and A. A. Starobinsky, Phys. Rev. D56, 3258 (1997), hep-ph/9704452.

[15] P. B. Greene, L. Kofman, A. D. Linde, and A. A. Starobinsky, Phys. Rev. D56 (1997) 6175, hep$\mathrm{ph} / 9705347$.

[16] An explicit construction for $\lambda \phi^{4}$ theory is given in: D. Kaiser, Phys. Rev. D57 (1998) 702

[17] N. W. MacLachlan, Theory and Application of Mathieu Functions, (Dover 1961).

[18] C. J. Hogan and M. J. Rees, Phys. Lett. B205 (1988) 228; E. Kolb and I. I. Tkachev, Phys. Rev. Lett. 71 (1993) 3051.

[19] E. Kolb \& I. I. Tkachev, Phys. Rev. D49 (1994) 5040; E. Kolb \& I. I. Tkachev, astro-ph/9510043

[20] R. Davis, Phys. Lett. B180, (1985) 225

[21] S. Chang, C. Hagmannn \& P. Sikivie, hep-ph/9807374

[22] P. B. Greene \& L. Kofman, hep-ph/9807339

[23] E. Ince, Ordinary Differential Equations, (Dover 1956). 